\section{How de novo mutation studies will change our view of the genetics of neurological and psychiatric disorders}
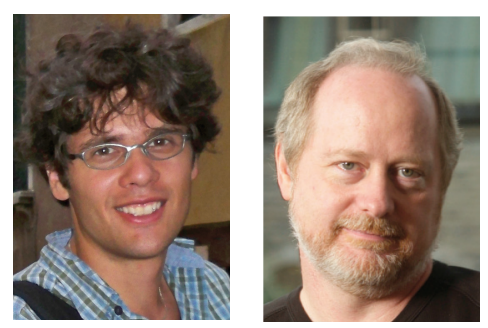

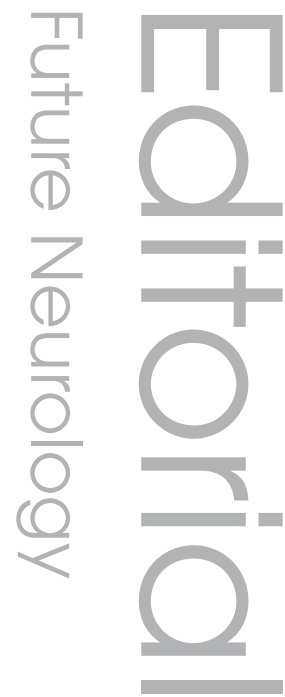

\section{Simon L Girard ${ }^{\dagger 1}$ \& Guy A Rouleau' \\ Université de Montréal, Centre Hospitalier Universitaire, Sainte-Justine Research Center, Montréal, Québec, Canada \\ Author for correspondence: simon.girard.3@umontreal.ca}

The search for the genetic etiology of brain diseases has been a constant priority for biomedical research in recent decades. While it has been very successful for specific diseases, particularly those for which a typical Mendelian transmission is observed, far less progress has been made for more common diseases where familial segregation is not as apparent. Nevertheless, both family aggregation and twin studies support the existence of a significant genetic contribution underlying the most common brain diseases. Recent technological advances provide the tools to begin to unravel the genetic mechanisms underlying complex disorders. Techniques such as genome-wide association studies (GWAS) and high-throughput sequencing now enable the testing of a broad genetic spectrum; before their advent, some genetic mechanisms could only be hypothesized, remaining untestable. Of particular interest to us and others has been the evaluation of the role of de novo mutations (DNMs; i.e., mutations arising sporadically during oogenesis or spermatogenesis) in brain diseases. With the development of targeted resequencing platforms, it is now possible to test the contribution of DNMs as a genetic mechanism for common brain diseases. Within the framework of this article, we will focus only on the small DNMs: mutations of a single or a few base pairs. Indeed, although large DNMs are important, they differ from their smaller counterparts in terms of their detection methods, frequencies and genetic roles; they will not be discussed in the present article.

The hypothesis that DNMs could be the cause of neurological or psychiatric disorders is not new. Indeed, one of the first studies to implicate a DNM with mental retardation dates back to the beginning of the 1990s [1]. However, at that time, large-scale mutational screening was not possible. Thus, by sequencing genes already associated with a disease, it was possible to detect DNMs present in the proband but absent from the parents. This is why for a rather long time DNMs were only detected in sporadic cases of diseases with a Mendelian transmission profile. For complex disorders, the identification of DNMs was almost impossible due to a lack of appropriate high-throughput sequencing methods. It was only with the completion of the Human Genome Project that large scale gene-screening endeavors became feasible. For instance, it became possible to sequence selected candidate genes (e.g., synaptic genes) in cohorts of trios (each trio consisting of a proband affected with schizophrenia and his/her parents) and generate lists of diseasespecific 'risk' genes bearing DNMs. Our group was among the first to use such a method in order to sequence a 1000 of these synaptic genes in a cohort of patients affected either with schizophrenia, autism or mental retardation (Synapse2-Disease [S2D] project). This study, followed by population screening studies, allowed us to identify DNMs responsible for schizophrenia $[2,3]$, autism [4] and mental retardation [5,6]. It is important to note that the sequencing costs of this study were in the order of CAN $\$ 10$ million. More recently, following the same trends, exome (i.e., all the protein-coding sequences of a genome)-sequencing studies have, in quick succession, led to the identification of multiples DNMs in the same three diseases. First, Vissers and coworkers have sequenced ten mental retardation trios in order to identify ten nonsynonymous DNMs, among which six are potentially

\section{Keywords}

- autism = exome sequencing = high-throughput sequencing

- mental retardation

- neurological disorder - psychiatric disorder - schizophrenia

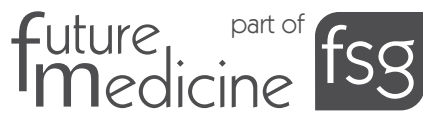


pathogenic [7]. Second, O'Roak and coworkers used a similar approach on 20 autism trios and identified ten nonsynonymous DNMs, including one frame-shift mutation [8]. Finally, our group has sequenced 14 schizophrenia trios and we found 15 nonsynonymous DNMs, of which four are nonsense mutations (introducing a premature stop codon) [9]. Remarkably, this last project sequenced four-times more DNA than the precedent S2D project for one 50th of the cost. An independent study later confirmed our findings in schizophrenia by identifying 40 DNMs in 53 trios [10]. Most interesting is their observed rate of nonsynonymous versus synonymous mutations $(16: 1)$. In the same vein, the 1000 Genomes Project (which aims at sequencing the complete genome of 2500 individuals) also identified DNMs in disease-free individuals, confirming that DNMs are sporadic events that happen naturally in a human genome [11]. According to these data, one could expect to find between zero and two DNMs in the exome of one individual. When considering studies from the three diseases mentioned earlier (mental retardation, schizophrenia and autism), it has been shown that the observed DNM frequency is significantly higher than what we would expect in normal human genomes of healthy individuals, suggesting that a fraction of these are likely disease predisposing $[6,9,12]$.

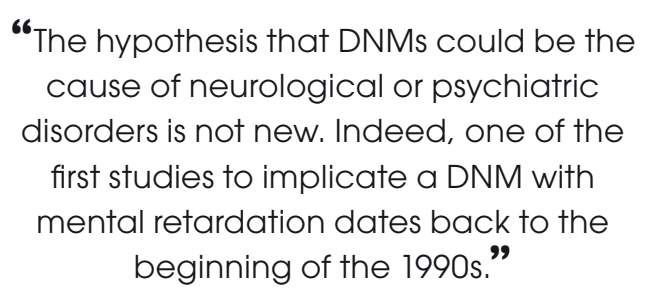

The issue of 'DNMs versus GWAS' is often raised. Indeed, it is often thought that the two concepts, common variants and rare variants, are a duality. This is not the case. In many instances, GWAS explain a significant fraction of the heritability of a disease, but in most situations, a large part is left unknown. Why can some of this large portion of heritablility not be explained by DNMs? Moreover, if common psychiatric and neurological diseases were only explained by common variants, any disease with a diminished reproductive fitness would be bound to have a decrease in its prevalence. However, for schizophrenia and many other diseases, a clear decrease in reproductive fitness has been observed [13], but no decrease in the prevalence has ever been reported. The explanation for this could be that new disease-causing alleles are continuously introduced into the genetic pool, replacing those lost due to reduced reproductive fitness; the occurrence of DNM is consistent with this hypothesis.

The high rates of DNMs observed in these three diseases beg the question: what is the role of DNM in other common neurological and psychiatric disorders, such as epilepsy, depression and Alzheimer's disease, among others? Although very few studies looking for DNMs have been conducted for these diseases, some exist for Alzheimer's disease. A few reports mention the potential implication of DNMs in early-onset Alzheimer's disease, particularly in the presenilin 1 gene [14,15]. Indeed, a critical factor in evaluating the potential role of DNM in any disease may in fact be age of onset. For diseases that do not affect reproductive fitness, and so begin after the age of procreation, DNMs may have a more limited role. If de novo alleles are not eliminated from the population via reduced reproductive fitness, one would expect that the disease frequency should rise in the population, which is not the case for most diseases.

In addition to providing a new genetic mechanism for many brain diseases, identifying DNMs provides a method for identifying new disease-predisposing candidate genes. However, as stated earlier, DNM are sporadic events occurring naturally in a genome. So the challenge is to distinguish pathogenic mutations from benign ones. We think that the answer resides in two words: 'numbers' and 'networks'. The previously cited studies were carried out on a fairly small number of probands (between 10 and 50). With such a small number, one must rely on very strong biological effects (e.g., protein-truncating variants) to enable the classification. However, if similar studies were to be made on a very large number of probands, (e.g., many hundreds) the number of identified DNMs would be very large. These genes could then be studied together bioinformatically using 'omics datasets (e.g., genomic, proteomic and transcriptomic, among others), looking for networks and patterns that link up certain genes into molecular machines. We predict that 'risk' genes for any particular disease will act on a limited number of pathways, all leading to similar biological outcomes. Such 'networks' could be formidable assets not only for the advancement of the clinical genetic diagnosis, but also for identifying currently unsuspected therapeutic targets and strategies.

The role of DNMs in brain diseases is only now being explored. DNMs will have important implications on diagnostic strategies and genetic counseling, and will also provide strategies to better understand and ultimately treat many common diseases. 


\section{Financial \& competing interests disclosure}

The authors have no relevant affiliations or financial involvement with any organization or entity with a financial interest in or financial conflict with the subject matter or materials discussed in the manuscript. This includes employment, consultancies, honoraria, stock ownership or options, expert testimony, grants or patents received or pending, or royalties.

No writing assistance was utilized in the production of this manuscript.

\section{Bibliography}

1. De Boulle K, Verkerk AJ, Reyniers E et al. A point mutation in the FMR-1 gene associated with fragile $\mathrm{X}$ mental retardation. Nat. Genet. 3(1), 31-35 (1993).

2. Gauthier J, Siddiqui TJ, Huashan P et al. Truncating mutations in NRXN2 and NRXN1 in autism spectrum disorders and schizophrenia. Hum. Genet. 130(4), 563-573 (2011).

3. Gauthier J, Champagne N, Lafreniere RG et al. De novo mutations in the gene encoding the synaptic scaffolding protein SHANK3 in patients ascertained for schizophrenia. Proc. Natl Acad. Sci. USA 107(17), 7863-7868 (2010).

4. Piton A, Michaud JL, Peng $\mathrm{H}$ et al. Mutations in the calcium-related gene ILIRAPLI are associated with autism. Hum. Mol. Genet. 17(24), 3965-3974 (2008).

5. Hamdan FF, Gauthier J, Spiegelman D et al. Mutations in SYNGAP1 in autosomal nonsyndromic mental retardation. $N$. Engl. J. Med.360(6), 599-605 (2009).

6. Hamdan FF, Gauthier J, Araki Y et al. Excess of de novo deleterious mutations in genes associated with glutamatergic systems in nonsyndromic intellectual disability. Am. J. Hum. Genet. 88(3), 306-316 (2011).

7. Vissers LE, de Ligt J, Gilissen C et al. A de novo paradigm for mental retardation. Nat. Genet. 42(12), 1109-1112 (2010).

8. O'Roak BJ, Deriziotis P, Lee C et al. Exome sequencing in sporadic autism spectrum disorders identifies severe de novo mutations. Nat. Genet. 43(6), 585-589 (2011).

9. Girard SL, Gauthier J, Noreau A et al. Increased exonic de novo mutation rate in individuals with schizophrenia. Nat. Genet. 43(9), 860-863 (2011).
10. Xu B, Roos JL, Dexheimer P et al. Exome sequencing supports a de novo mutational paradigm for schizophrenia. Nat. Genet. 43(9), 864-468 (2011).

11. Durbin RM, Abecasis GR, Altshuler DL et al. A map of human genome variation from population-scale sequencing. Nature 467(7319), 1061-1073 (2010).

12. Awadalla P, Gauthier J, Myers RA et al. Direct measure of the de novo mutation rate in autism and schizophrenia cohorts. Am. J. Hum. Genet. 87(3), 316-324 (2010).

13. MacCabe JH, Koupil I, Leon DA. Lifetime reproductive output over two generations in patients with psychosis and their unaffected siblings: the Uppsala 1915-1929 birth cohort multigenerational study. Psycholog. Med. 39(10), 1667-1676 (2009).

14. Golan MP, Styczynska M, Józwiak K et al. Early-onset Alzheimer's disease with a de novo mutation in the presenilin 1 gene. Exp. Neurol. 208(2), 264-268 (2007).

15. Portet F, Dauvilliers Y, Campion D et al. Very early onset $\mathrm{AD}$ with a de novo mutation in the presenilin 1 gene (Met $233 \mathrm{Leu})$. Neurology 61(8), 1136-1137 (2003). 